\title{
Long-term outcomes of patients with systemic lupus erythematosus: A Multicenter Cohort Study from CSTAR registry
}

Ziqian Wang ${ }^{1, \dagger}$, Mengtao Li $i^{1, \dagger}$, Zhizhong Ye ${ }^{2, \dagger}$, Caifeng $\mathrm{Li}^{3}$, Zhijun $\mathrm{Li}^{4}$, Xiangpei $\mathrm{Li}^{5}$, Lijun $\mathrm{Wu}^{6}$, Shengyun $\mathrm{Liu}^{7}$, Xiaoxia Zuo Ping Zhu ${ }^{9}$, Zhengang Wang ${ }^{10}$, Li Ma ${ }^{11}$, Miaojia Zhang ${ }^{12}$, Zhiduo Hou ${ }^{13}$, Liqi Bi ${ }^{14}$, Yanhong Wang ${ }^{15}$, Jiuliang Zhao ${ }^{1}$, Qian Wang ${ }^{1}$, Xinping $\operatorname{Tian}^{1}$, Xiaofeng Zeng ${ }^{1, *}$; CSTAR co-authors

${ }^{1}$ Department of Rheumatology, Peking Union Medical College Hospital, Peking Union Medical College and Chinese Academy of Medical Sciences, Key Laboratory of Rheumatology and Clinical Immunology, Ministry of Education, Beijing, China

${ }^{2}$ Department of Rheumatology, The Fourth People's Hospital of Shenzhen Affiliated to Guangdong Medical College, Shenzhen, China

${ }^{3}$ Department of Rheumatology, Beijing Children Hospital Affiliated to Capital Medical University, Beijing, China

${ }^{4}$ Department of Rheumatology, The Affiliated Hospital of Bengbu Medical College, Bengbu, China

${ }^{5}$ Department of Rheumatology, The First Affiliated Hospital of USTC, Anhui Provincial Hospital, Hefei, China

${ }^{6}$ Department of Rheumatology, The People's Hospital of Xinjiang Autonomous Region, Urumqi, China

${ }^{7}$ Department of Rheumatology, The First Affiliated Hospital of Zhengzhou University, Zhengzhou, China

${ }^{8}$ Department of Rheumatology, Xiangya Hospital, Central South University, Changsha, China

${ }^{9}$ Department of Clinical Immunology, PLA Specialized Research Institute of Rheumatology and Immunology, Xijing Hospital, Airforce Military Medical University, Xi'an, China

${ }^{10}$ Department of Rheumatology, Beijing Tongren Hospital Affiliated to Capital Medical University, Beijing, China

${ }^{11}$ Department of Rheumatology, China-Japan Friendship Hospital Affiliated to the National Health Commission of PRC, Beijing, China

${ }^{12}$ Department of Rheumatology, The First Affiliated Hospital of Nanjing Medical University, Nanjing, China

${ }^{13}$ Department of Rheumatology, The First Affiliated Hospital of Shantou University Medical College, Shantou, China

${ }^{14}$ Department of Rheumatology, Sino-Japanese friendship Hospital of Jilin University, Changchun, China

${ }^{15}$ Department of Epidemiology and Bio-statistics, Institute of Basic Medical Sciences, China Academy of Medical Sciences and Peking Union

Medical College, Beijing, China

Abstract

Received February 10, 2021 accepted October 12, 2021

Objective: To study the long-term outcomes, in the context of both mortality and organ damage in patients with systemic lupus erythematosus (SLE) in the Chinese SLE Treatment and Research group (CSTAR) registry cohort.

Methods: Patients were enrolled from April 2009 to February 2010 and they were followed up. The demographic data, clinical manifestations, labs test results and imaging examinations, disease activity (SLEDAI-2K), damage scores (SLLIC/ Damage Index [SDI]), and medications were collected. Data were censored at either the last clinic visit or telephonic interview. Survival rate was analyzed by Kaplan-Meier (KM) method. COX proportional hazard model was adopted to perform the analysis of predicting factors for mortality and organ damage. Logistic regression analysis was employed to discuss the relationship among mortality, organ damage, and flare.

Results: A total of 2104 patients were recruited at baseline and 1494 patients were followed up. The cumulative 1-year, 3-year, and 5-year survival rates were $98.3 \%, 96.9 \%$, and $95.7 \%$, respectively. Seventy-eight patients died during follow-up, and the main causes of death were infection $(34.6 \%)$, active disease $(26.9 \%)$, cardiovascular and cerebrovascular events $(5.13 \%)$, and malignancy $(5.13 \%)$. At entry, 247 patients presented with irreversible organ damage and it increased to 398 patients at the endpoint. The major accumulated organ damages were kidney $(25.9 \%)$, musculoskeletal disease $(20.2 \%)$, neuropsychiatric disease $(12.2 \%)$, and pulmonary damage $(10.9 \%)$. Cox regression analysis further showed that male, late disease onset, delayed diagnosis (diagnosis from disease onset $>1$ year), baseline organ damage, and specific organ involvements predicted for higher mortality. In addition, early disease onset was a protecting factor for organ damage, and anti-SSA was an independent predicting factor for new organ damage. Logistic regression analysis showed that flare predicted for more organ damage.

Conclusion: The 5-year survival rate of Chinese SLE patients has improved and is comparable to Caucasians SLE patients. Disease flare impact on prognosis is the increasing risk of damage development. Early diagnosis, prevention for flare and damage to maintain remission, may improve outcome.

Keywords

systemic lupus erythematosus Flare $\bullet$ organ damage $•$ prognosis

†Ziqian Wang, Mengtao Li, and Zhizhong Ye contributed equally to this study.

\section{Address for correspondence:}

*Xiaofeng Zeng, Department of Rheumatology, Peking Union Medical College

Hospital, No. 1 Shuaifuyuan, Wangfujing Avenue, Beijing 100730, China

E-mail: zengxfpumc@163.com 
Increasing number of cohort studies have shown that the long-term survival of systemic lupus erythematosus (SLE), a multi-organ involvement systemic autoimmune disease, has been greatly improved during last decades. ${ }^{[1-3]}$ Among the factors that might impact the survival rate, organ damage was proposed to have great influence on long-term prognosis of SLE. ${ }^{[4]}$ However, there are only few large sample-size studies related to the prognosis of SLE patients, especially for Chinese SLE patients, including long-term survival, predicting factors, organ damage and flare, etc. ${ }^{[5-10]}$ Therefore, we are in need of more research to move further in prognostic studies for SLE.

This study was aimed to explore long-term outcomes of Chinese SLE patients, including mortality and organ damage accrual. CSTAR registry cohort is the first multi-center registry cohort across the whole country, so it can represent the real situation of the country.

\section{Patients and Methods}

\section{Patient recruitment and follow-up}

CSTAR registry cohort is the first multi-center registry cohort in China. A total of 104 high-ranked rheumatology centers from 30 different provinces all over China participated in the registry. In our study, patients registered during April 2009 to February 2010 were considered. The baseline data and related analysis of this cohort were published from 2013 to 2015. ${ }^{[11-16]}$

All patients involved in this study signed the informed consent form, either by themselves or their guardians. ${ }^{[16]}$ The inclusion criterion was Chinese with SLE who fulfilled the 1997 revised American College of Rheumatology (ACR) criteria. All enrolled patients were followed up regularly or at least once a year and were treated according to their condition.

This study was approved by the Ethics Committee of $\mathrm{PUMCH}$, Chinese Academy of Medical Sciences (approval number: S-197).

\section{Definitions of organ damage, organ involvement, and flare}

Organ damage was defined as a specific damage resulted in organ dysfunction and increased morbidity. ${ }^{[17]}$ In our cohort, damage was evaluated using Systemic Lupus International Collaborating Clinics (SLICC) damage index (SDI). ${ }^{[1]}$

According to 2012 SLICC criteria and 2019 EULAR criteria for SLE, ${ }^{[19,20]}$ organ involvements were defined as: (1) Lupus nephritis (LN): $24-\mathrm{h}$ urine protein $>500 \mathrm{mg} / 24 \mathrm{~h}$ or equivalent spot urine protein-to-creatinine ratio or red blood cell casts or proved by pathology; (2) Neuropsychiatric lupus erythematosus (NPLE): seizures, psychosis, mononeuritis multiplex, myelitis, peripheral or cranial neuropathy, acute confusion state in the absence of other known causes; (3) Hematologic involvement (HEMA): hemolytic anemia and/or thrombocytopenia with/without leukopenia; (4) Serositis (SERO): typical pleurisy or pleural effusions or pleural rub, typical pericardial pain or pericardial effusion or pericardial rub or pericarditis by electrocardiography; (5) Interstitial Lung Disease (ILD) caused by Lupus; (6) Pulmonary arterial hypertension (PAH), confirmed by echocardiogram or catheterization and ascertained that was secondary to SLE. ${ }^{[21,22]}$

Flare was defined as increase of SLE disease activity (SLEDAI-2K) to $\geq 4$ points in last 4 weeks (including at least 1 point of the non-serological items [except for complement and anti-dsDNA]) or appearing of a new SLE manifestation or worsening of pre-existing manifestation (whether in SLEDAI$2 \mathrm{~K}$ or not) that require strengthening immunosuppressive therapy (including restarting or increasing steroids or immunosuppressants). ${ }^{[23]}$

\section{Data collection}

We extracted baseline data from CSTAR online registry, including demographic, clinical presentations, laboratory and imaging test results, and SLEDAI-2K and SDI scores. Electronic medical records were reviewed to collect follow-up data, mainly for flare-related information, SDI scores, death time, and causes. Telephonic interview was employed to supplement additional data for analysis.

\section{Statistical analysis}

SPSS 19.0 was applied to perform the statistical analysis. Kaplan-Meier (KM) curve was obtained to gain 1-year, 3-year, and 5-year survival rates. Cox regression analysis was applied to study potential predicting factors for death and organ damage. The results were presented as hazard rate (HR) and $95 \%$ confidence intervals (Cls). Univariate cox regression was first done to search for potential factors. Identified factors in univariate analysis were used to discover independent predicting factors in multivariate analysis. All the factors involved in our study were binary variables; chi-squared test was performed first to test the association among the identified factors in univariate analysis before multivariate analysis was conducted. Descriptive analysis was performed to display the development of organ damage and disease flares. Moreover, logistic regression model was adopted to further explore the correlation among mortality, organ damage and flare, showing the results as risk ratio $(R R)$ and $95 \% \mathrm{Cls}$.

\section{Results}

\section{Baseline characteristics}

At entry, 2104 SLE patients from different clinical centers all around China were recruited for the registry cohort. Finally, 
1494 patients who had the required follow-up information and had been followed-up for mean time of $5.43 \pm 1.82$ years were included in this study. The baseline average age of disease onset was $28.9 \pm 12.2$ years old $(\mathrm{yd})$, the mean age at diagnosis was $29.8 \pm 12.4 \mathrm{yd}$, and the average duration of diagnosis delay was $1.14 \pm 2.55$ years. Patients who were lost to follow-up were excluded from the final analysis. The baseline clinical manifestations and organ involvements of the 1494 patients who were successfully followed up were malar rash $(47.5 \%)$, discoid lesions $(6.22 \%)$, photosensitivity $(23.5 \%)$, oral ulcers $(21.9 \%)$, arthritis $(54.1 \%)$, SERO (20.5\%), HEMA (56.6\%), LN (57.3\%), NPSLE (7.63\%), ILD (4.48\%), and PAH (4.02\%).

Baseline comparison was employed to study the difference between the study group and the lost to follow-up group, including demographics, clinical manifestations, and autoantibody profiles. The disease duration and time from onset to diagnosis were comparable between these two groups, whereas the mean onset age of the lost to follow-up group was higher than the study group as $30.9 \pm 12.19 \mathrm{yd}(P<0.001)$. For clinical manifestations, no significant differences were identified except for photosensitivity (6.379, $P=0.012)$, which was not extremely specific or closely related to SLEDAI-2K and damage accrual. The lost to follow-up group had higher proportion of anti-SM antibody (17.9\% vs. $13.3 \%, P=0.009)$ and anti-RNP antibody (10.6\% vs. $4.75 \%, P<0.001)$, and it was found that there had not been any significant difference in other autoantibodies, including anti-dsDNA, anti-SSA, anti-SSB, anti-rRNP, and antiphospholipid (APL).

\section{Long-term survival and causes of death}

The overall 1-year, 3-year, and 5-year survival rates from enrollment were $98.3 \%, 96.9 \%$, and $95.7 \%$, respectively (Figure 1). During follow-up, 78 patients died for different causes: infection ( $n=27,34.6 \%)$, active disease $(n=21$, $26.9 \%)$, malignancy ( $n=4,5.13 \%)$, cardiovascular event ( $n=2,2.56 \%$ ), cerebrovascular event $(n=2,2.56 \%$ ), and undetermined reasons. Considering infection, 56\% $(n=15)$ of them were found to be pulmonary infection. Central nervous system infection and blood stream infection found to be $22 \%(n=6)$ and 15\% $(n=4)$, respectively. Considering patients who died of active disease, $38 \%(n=8)$ of the deaths

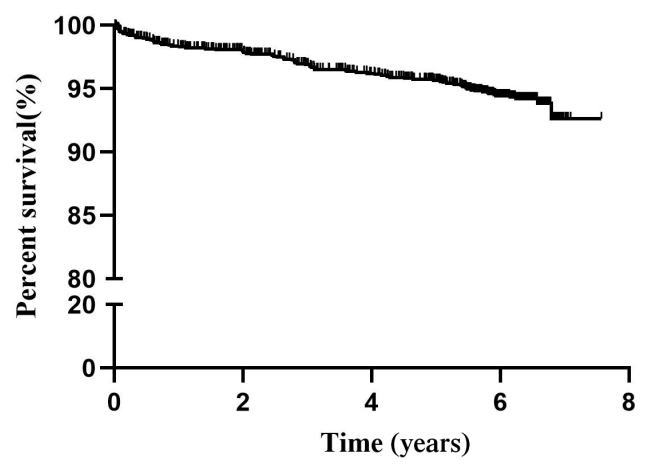

Figure 1: Kaplan-Meier survival curve of the whole cohort.

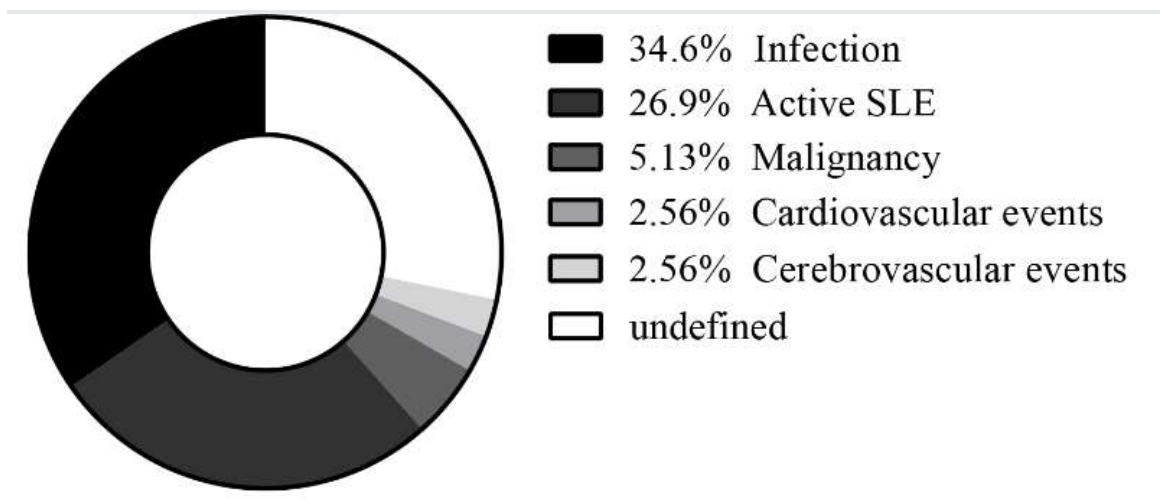

Figure 2: Proportion of different death causes. 
were caused by renal failure. Another 19\% $(n=4)$ of the deaths were caused by NPLE, and the rest were PAH $(n=3$, $14 \%)$, HEMA ( $n=2,10 \%)$, ILD ( $n=2,10 \%)$, gastrointestinal disease $(n=1,5 \%)$, and diffuse alveolar hemorrhage $(n=1$, $5 \%$ ) (Figure 2).

\section{Accumulation of organ damage and flare}

In our cohort, 247 patients had organ damage at baseline. A total of 191 patients developed new damage during followup. At the endpoint, 398 patients developed organ damage with a total of 525 accumulated damages. The top five organ damages were renal (25.9\%), musculoskeletal (20.2\%), neuropsychiatric $(12.2 \%)$, pulmonary $(10.9 \%)$, and ocular damage $(7.4 \%)$ (Figure 3). During follow-up, a total of 625 patients $(41.8 \%)$ experienced at least one episode of flare. The median interval was $2.0 \pm 1.9$ years for the first flare after enrollment.

\section{Prediction of mortality}

In univariate analysis, we found that male (HR 1.903, 95\% Cl 1.029-3.522, $P=0.040$ ), disease onset age $>50$ years (HR 3.339, 95\% Cl 1.786-6.243, $P<0.001$ ), disease onset to diagnosis $>1$ year (HR 1.620, 95\% Cl 1.020-2.573, $P=0.041)$, baseline organ damage $(\mathrm{HR} 2.594,95 \% \mathrm{Cl}$ 1.620-4.153, $P<0.001)$, LN (HR 2.323, 95\% Cl 1.444$3.738, P=0.001)$, NPLE (HR 2.539, 95\% Cl 1.267-5.087, $P=0.009), \mathrm{PAH}(\mathrm{HR} 3.693,95 \% \mathrm{Cl} 1.901-7.173, P<0.001)$, and SERO (HR 2.090, 95\% Cl 1.276-3.422, $P=0.003$ ) were shown to be potential predicting factors for mortality (Table 1). Chi-squared test showed that SERO was positively related with baseline damage $(8.835, P=0.003), \mathrm{LN}$ (46.902, $P<0.001)$, and PAH (54.663, $P<0.001)$, and baseline damage was also positively associated with $\mathrm{LN}(24.732$, $P<0.001)$, PAH (28.616, $P<0.001)$, and NPLE (40.145, $P<0.001)$. Therefore, SERO and baseline damage might reduce the prediction power and should be considered to be excluded if they compromise the prediction power of other factors in multivariate analysis. However, they were kept and used in final multivariate analysis because the prediction effect was not influenced significantly even if they were included in the final equation. In multivariate analysis, it was confirmed that onset age $>50$ years $(\mathrm{HR} 3.347,95 \% \mathrm{Cl}$ 1.781-6.287, $P<0.001$ ), disease onset to diagnosis $>1$ year (HR 1.604, 95\% Cl 1.006-2.558, $P=0.047$ ), LN (HR 2.055, $95 \% \mathrm{Cl} 1.264-3.341, P=0.004)$, NPLE (HR 2.550, 95\% Cl 1.252-5.196, $P=0.010)$, PAH (HR 2.400, 95\% Cl 1.170$4.920, P=0.017$ ), and baseline organ damage (HR 1.907, $95 \% \mathrm{Cl} 1.169-3.110, P=0.010)$ were independent factors for prediction of mortality (Table 1 ).

Regarding organ damage, onset age $>50$ years (HR 2.048, $95 \% \mathrm{Cl} 1.285-3.264, P=0.003)$, baseline organ damages (HR 1.434, 95\% Cl 1.012-2.032, $P=0.043$ ), anti-SSA (HR 1.355, 95\% Cl 0.995-1.844, $P=0.054)$, anti-SSB (HR $1.458,95 \% \mathrm{Cl} 0.982-2.165, P=0.061)$ and anti-rRNP (HR $1.553,95 \% \mathrm{Cl} 0.977-2.468, P=0.063)$ were shown to be potential risk factors for damage accrual in univariate analysis. In addition, onset age $<18$ years (HR 0.484, 95\% $\mathrm{Cl} 0.293-0.799, P=0.005$ ) was identified as a protecting factor for new damage. As for multivariate analysis, factors with $P$-value $<0.1$ were candidates. Among them, antirRNP was excluded as testing data were missing for half of the patients at entry. The chi-squared test was performed to evaluate their correlation. Positive relation was shown as below: PAH and baseline damage (28.616, $P<0.001)$, $\mathrm{PAH}$ and anti-SSA antibody $(4.814, P=0.028)$, ILD and baseline damage (11.152, $P=0.001)$, ILD and disease onset age $>50$ years $(3.973, P=0.046)$, and anti-SSA and anti-SSB antibody (308.575, $P<0.001)$. According to the correlation study, onset age $>50$ years, baseline organ damage, PAH, ILD, and anti-SSA antibody were finally included in multivariate analysis, because anti-SSB antibody was nearly overlapped with anti-SSA antibody. Late-onset age and anti-SSA antibody still showed their prediction power for organ damage even with the compromise impact of other positively related factors and were identified as potential independent predicting factors for new organ damage (Table 2).

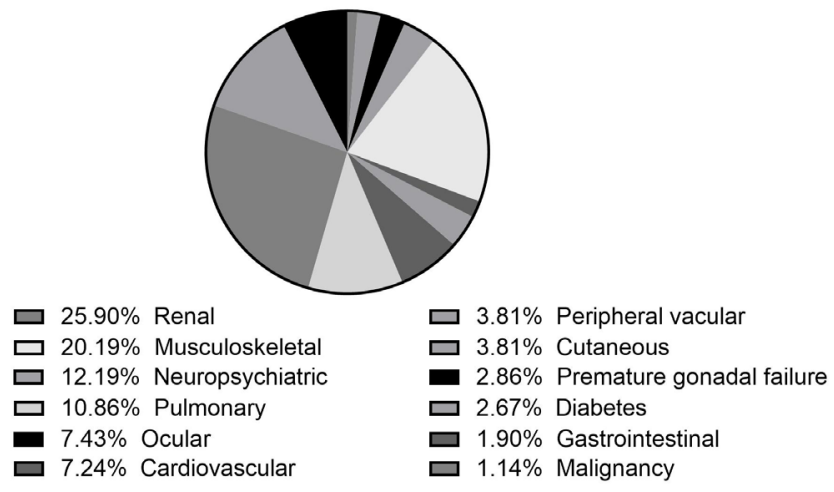

Figure 3: Distribution of different organ damages. 
Table 1: Univariate and multivariate COX regression analyses for mortality

\begin{tabular}{|c|c|c|c|}
\hline Univariate analysis & HR & $\mathbf{9 5 \%} \mathrm{Cl}$ & $\boldsymbol{P}$ \\
\hline Male & 1.903 & $1.029-3.522$ & 0.040 \\
\hline Onset age $>50$ years & 3.339 & $1.786-6.243$ & $<0.001$ \\
\hline Onset age $<18$ years & 1.001 & $0.535-1.872$ & 0.998 \\
\hline $\begin{array}{l}\text { Onset to diagnosis } \\
>1 \text { year }\end{array}$ & 1.620 & $1.020-2.573$ & 0.041 \\
\hline Baseline organ damage & 2.594 & $1.620-4.153$ & $<0.001$ \\
\hline LN & 2.323 & $1.444-3.738$ & 0.001 \\
\hline HEMA & 1.310 & $0.825-2.081$ & 0.252 \\
\hline ILD & 1.828 & 0.79504 .206 & 0.156 \\
\hline NPLE & 2.539 & $1.267-5.087$ & 0.009 \\
\hline $\mathrm{PAH}$ & 3.693 & $1.901-7.173$ & $<0.001$ \\
\hline SERO & 2.090 & $1.276-3.422$ & 0.003 \\
\hline Anti-dsDNA & 0.769 & $0.492-1.202$ & 0.249 \\
\hline Anti-SM & 1.001 & $0.561-1.785$ & 0.997 \\
\hline Anti-RNP & 1.512 & $0.817-2.798$ & 0.188 \\
\hline Anti-SSA & 1.411 & $0.877-2.271$ & 0.156 \\
\hline Anti-SSB & 1.302 & $0.688-2.464$ & 0.417 \\
\hline Anti-rRNP & 0.951 & $0.429-2.108$ & 0.901 \\
\hline APL & 1.150 & $0.581-2.277$ & 0.688 \\
\hline Low completement & 0.958 & $0.603-1.522$ & 0.856 \\
\hline Multivariate analysis & HR & $95 \% \mathrm{Cl}$ & $P$ \\
\hline Male & 1.747 & $0.936-3.262$ & 0.080 \\
\hline Onset age $>50$ years & 3.347 & $1.781-6.287$ & $<0.001$ \\
\hline $\begin{array}{l}\text { Onset to diagnosis } \\
>1 \text { year }\end{array}$ & 1.604 & $1.006-2.558$ & 0.047 \\
\hline LN & 2.055 & $1.264-3.341$ & 0.004 \\
\hline NPLE & 2.550 & $1.252-5.196$ & 0.010 \\
\hline $\mathrm{PAH}$ & 2.400 & $1.170-4.920$ & 0.017 \\
\hline SERO & 1.575 & $0.938-2.646$ & 0.086 \\
\hline Baseline organ damage & 1.907 & $1.169-3.110$ & 0.010 \\
\hline
\end{tabular}

HEMA, hematologic involvement; ILD, interstitial lung disease; LN, lupus nephritis; PAH, pulmonary arterial hypertension; SERO, serositis.

\section{Flare to organ damage and mortality}

Previously, it was proved that pre-existing damage predicts for more damage and death. Univariate logistic model was applied to analyze the predicting effect of past flares to new damage and mortality. Flare was shown to predict for more organ damage (RR 1.831, 95\% Cl 1.343-2.496, $P<0.001$; onset age, baseline damage, PAH, ILD, anti-SSA, and anti-SSB were all controlled for analysis). However, no direct prediction effect was found for flare to mortality (RR $1.042,95 \% \mathrm{Cl} 0.647-1.679, P=0.864$; the controlled factors were gender, onset age, baseline damage, $P A H, L N$, and NPLE).
Table 2: Univariate and multivariate COX regression analyses for organ damage

\begin{tabular}{|c|c|c|c|}
\hline Univariate analysis & HR & $95 \% \mathrm{Cl}$ & $P$ \\
\hline Male & 1.394 & $0.894-2.173$ & 0.143 \\
\hline Onset age $>50$ years & 2.048 & $1.285-3.264$ & 0.003 \\
\hline Onset age $<18$ years & 0.484 & $0.293-0.799$ & 0.005 \\
\hline Onset to diagnosis $>1$ year & 0.804 & $0.571-1.133$ & 0.213 \\
\hline Baseline organ damage & 1.434 & $1.012-2.032$ & 0.043 \\
\hline LN & 1.118 & $0.842-1.485$ & 0.440 \\
\hline HEMA & 0.939 & $0.706-1.250$ & 0.668 \\
\hline ILD & 1.707 & $0.972-2.999$ & 0.063 \\
\hline NPLE & 1.486 & $0.846-2.610$ & 0.168 \\
\hline PAH & 1.763 & $0.982-3.163$ & 0.057 \\
\hline SERO & 1.178 & $0.817-1.700$ & 0.380 \\
\hline Anti-dsDNA & 1.179 & $0.883-1.574$ & 0.265 \\
\hline Anti-SM & 1.116 & $0.779-1.597$ & 0.550 \\
\hline Anti-RNP & 1.396 & $0.929-2.097$ & 0.108 \\
\hline Anti-SSA & 1.355 & $0.995-1.844$ & 0.054 \\
\hline Anti-SSB & 1.458 & $0.982-2.165$ & 0.061 \\
\hline Anti-rRNP & 1.553 & $0.977-2.468$ & 0.063 \\
\hline APL & 1.195 & $0.792-1.803$ & 0.395 \\
\hline Low completement & 0.993 & $0.738-1.337$ & 0.965 \\
\hline Multivariate analysis & HR & $95 \% \mathrm{Cl}$ & $P$ \\
\hline Onset age $>50$ years & 2.258 & $1.415-3.603$ & 0.001 \\
\hline Baseline organ damage & 1.378 & $0.967-1.963$ & 0.076 \\
\hline ILD & 1.583 & $0.896-2.795$ & 0.114 \\
\hline $\mathrm{PAH}$ & 1.584 & $0.874-2.872$ & 0.130 \\
\hline Anti-SSA & 1.396 & $1.024-1.904$ & 0.035 \\
\hline
\end{tabular}

HEMA, hematologic involvement; ILD, interstitial lung disease; LN, lupus nephritis; PAH, pulmonary arterial hypertension; SERO, serositis.

\section{Discussion}

Compared with a Bayesian meta-analysis performed by Tektonidou et al. ${ }^{[10]}$ in 2017, the 5-year survival rate in our study was comparable to the reported survival rate in highincome countries (95.7\% vs. 95\%). Our study obtained relatively higher long-term survival rates compared with the result of our previous meta-analysis for Chinese SLE patients as well $(95.7 \%$ vs. $94 \%) .{ }^{[5]}$ For the causes of death, most of them were consistent with our previous meta-analysis except malignancy. The proportion of malignancy was relatively higher in this study than previous studies; this might be due to longer survival-time. SLE patients tend to develop malignancy because long-time employment of immunosuppressive agents. ${ }^{[24,25]}$

Several potential predicting factors for mortality were identified in this study, which included male gender, late disease onset, delayed diagnosis (the interval from symptom onset 
to diagnosis $>1$ year), baseline organ damage, LN, PAH, and NPLE. Compared with the results of our previous single-center study, with larger sample size (1494 vs. 260), we further detected that male gender, late-onset age, and specific organ involvements lead to more deaths ${ }^{[26]}$ Apart from those organ involvements that were reported to have worse prognosis, including LN, NPLE, and PAH; SERO was also shown to predict for higher mortality. According to our past study on SERO, SERO was closely associated with $L N$ and $\mathrm{PAH}$, which was consistent with our correlation analysis and might explain the predictive effect of SERO for mortality. ${ }^{[27]}$ Therefore, aggressive treatment for patients with LN, NPLE, and $\mathrm{PAH}$ to maintain persistent remission and reduce flare could be a possible strategy to obtain better prognosis.

Late age for disease onset was proven to be a predicting factor for damage, while early-onset was a protecting factor for damage, which was in accordance with our previous single-center study. ${ }^{[26]}$ In line with our study, previous study by Appenzeller et al. ${ }^{[28]}$ presented greater damage accrual for late-onset SLE patients, and Kim et al. ${ }^{[29]}$ found higher damage scores in adult-onset SLE than childhood-onset SLE with the same disease in the same duration strata. Anti-SSA antibody was also identified as a predicting factor for organ damage even after controlling other confounding factors, which was not reported in previous study. In our study, a total of 369 patients $(24.7 \%)$ had positive anti-SSA at baseline. Among them, 58 patients $(30.4 \%$ ) of the new damage group (a total of 191 patients) showed positive anti-SSA at entry. Mikdashi et al. ${ }^{[30]}$ in 2004 reported that anti-SSA antibody predicted for neuropsychiatric damage. Another article by Higuera-Ortiz et al. ${ }^{[3]]}$ proposed that anti-SSA antibody might be pathologically involved in lupus-associated mitral valve regurgitation. However, the real role of anti-SSA antibody in damage should be further elaborated by future studies.

Organ damage has been repeatedly reported to be a predictor for mortality in Caucasians cohorts and was proposed that it was driven by disease flare. ${ }^{[32-34]}$ By regression analysis among mortality, organ damage and flare, our study demonstrated again that disease flare predisposed SLE patients to more damage accrual and damage might further increase the chance of new damage, leading to more deaths in the end. Moreover, $22.4 \%$ of the damages in our study were associated with steroids treatment, including cataract, osteoporosis-related fracture, avascular necrosis, and diabetes mellitus, which might be the result of strengthening measures to control disease flares. Accordingly, we need to find measures to prevent disease flares with glucocorticoids sparing approach to improve prognosis, in other words, to keep patients in persistent remission with the lowest possible dose of glucocorticoids.

The major differences at baseline between the lost to follow-up group with the study group were older onset age, higher proportions of photosensitivity, and the presence of anti-SM and antiRNP antibodies. Among them, only the onset age was related to prognosis, and it was shown that late onset was a predicting factor for both mortality and organ damage. The proportion of late-onset patient was slightly lower in the study group (5.69\% vs. $7.21 \%)$. However, chi-squared test did not show statistically significant difference between these two groups $(P=0.068)$. Therefore, these two groups were roughly comparable at baseline, and the missing data during follow-up of the lost to followup group would not have great impact on our prognostic study.

The major limitation of this study is that the rate of lost to follow-up rate in our study was $29.0 \%$. Although it was basically comparable to the study group, the missing data would still lead to inaccuracy to the prognostic study. In China, it is hard to keep regular follow-up, because patients often go to remote and different hospitals to seek for medical service. In this study, due to partial and retrospective review of related data, part of the follow-up data was incomplete. In addition, we could not avoid the influence of disease course or other potential confounding factors on the analysis of longterm survival rates. Therefore, we need to conduct more prospective inception cohorts to verify our results.

\section{Conclusion}

The 5-year survival rate of Chinese SLE patients is comparable with those reported from Caucasians patients. Disease flare may lead to more damage and thus adversely impact long-term prognosis. After all, early diagnosis, taking measures to avoid or prevent flare, prevention for organ damage accrual, and focusing on specific organ involvements to achieve persistent remission may reduce mortality in the long-term and could be the treating target in the management of SLE patients.

Informed Consent

All patients involved in this study signed the informed consent form, either by themselves or their guardians.

Ethical approval

This study was approved by the Ethics Committee of PUMCH, Chinese Academy of Medical Sciences (approval number: S-197). 
Funding

This study was supported by the Chinese National Key Research R\&D Program (2017YFC0907601, 2017YFC0907602, and 2017YFC0907603), the Chinese National High Technology Research and Development Program, Ministry of Science and Technology (2012AA02A513), and the Chinese National Key Technology R\&D Program, Ministry of Science and Technology (2008BAI59B02).

Conflict of Interest

Xiaofeng Zeng is the Editor-in-Chief of the journal; Xinping Tian is the Executive Editor-in-Chief; Mengtao Li is an Associate Editor-in-Chief; Yanhong Wang and Jiuliang Zhao are Editorial Board Members. The article was subject to the journal's standard procedures, with peer review handled independently of these members and their research groups.

\section{References}

[1] Mak A, Cheung MW, Chiew HJ, et al. Global Trend of Survival and Damage of Systemic Lupus Erythematosus: Meta-analysis and Meta-regression of Observational Studies from the 1950s to 2000 s. Semin Arthritis Rheum, 2012;41(6):830-839.

[2] Rees F, Doherty M, Grainge MJ, et al. Mortality in Systemic Lupus Erythematosus in the United Kingdom 1999-2012. Rheumatology, 2016;55(5):854-860.

[3] Ugarte A, Ruiz-Irastorza G. SLE: The Changing Prognosis. Lupus, 2016;25(12):1285-1287.

[4] Pego-Reigosa JM, Lois-Iglesias A, Rua-Figueroa I, et al. Relationship Between Damage Clustering and Mortality in Systemic Lupus Erythematosus in Early and Late Stages of the Disease: Cluster Analyses in a Large Cohort from the Spanish Society of Rheumatology Lupus Registry. Rheumatology, 2016;55(7):1243-1250.

[5] Wang Z, Wang Y, Zhu R, et al. Long-term Survival and Death Causes of Systemic Lupus Erythematosus in China: A Systemic Review of Observational Studies. Medicine, 2015;94(17):e794.

[6] Feng X, Pan W, Liu L, et al. Prognosis for Hospitalized Patients with Systemic Lupus Erythematosus in China: 5-Year Update of the Jiangsu Cohort. PloS one, 2016;11(12):e0168619.

[7] Mu L, Hao Y, Fan Y, et al. Mortality and Prognostic Factors in Chinese Patients with Systemic Lupus Erythematosus. Lupus. 2018;27(10):1742-1752.

[8] Wang ZR, Ren LM, Li R, et al. Analysis of 20-year Survival Rate and Prognostic Indicators of Systemic Lupus Erythematosus. Zhonghua yi xue za zhi. 2019;99(3):178-182.

[9] Wu XY, Yang M, Xie YS, et al. Causes of Death in Hospitalized Patients with Systemic Lupus Erythematosus: A 10-year Multicenter Nationwide Chinese Cohort. Clin Rheumatol, 2019;38(1):107-115.

[10] Tektonidou MG, Lewandowski LB, Hu J, et al. Survival in Adults and Children with Systemic Lupus Erythematosus: A Systematic Review and Bayesian Meta-analysis of Studies from 1950 to 2016. Ann Rheum Dis, 2017;76(12):2009-2016.

[11] Zhang S, Su J, Li X, et al. Chinese SLE Treatment and Research group (CSTAR) Registry: V. Gender Impact on Chinese Patients with Systemic Lupus Erythematosus. Lupus, 2015;24(12):1267-1275.

[12] Xu D, You X, Wang Z, et al. Chinese Systemic Lupus Erythematosus Treatment and Research Group Registry VI: Effect of Cigarette Smoking on the Clinical Phenotype of Chinese Patients with Systemic Lupus Erythematosus. PloS One, 2015;10(8):e0134451.
[13] Tian X, Li M, Ye Z, et al. Related Factors of Fetal Loss in Chinese Women with Systemic Lupus Erythematosus: Data from Chinese SLE Treatment and Research Group Registry IV. Int J Rheum Dis, 2015;18(6):654-660.

[14] Li M, Wang Q, Zhao J, et al. Chinese SLE Treatment and Research Group (CSTAR) Registry: II. Prevalence and Risk Factors of Pulmonary Arterial Hypertension in Chinese Patients with Systemic Lupus Erythematosus. Lupus, 2014;23(10):1085-1091.

[15] Li J, Leng X, Li Z, et al. Chinese SLE Treatment and Research Group Registry: III. Association of Autoantibodies with Clinical Manifestations in Chinese Patients with Systemic Lupus Erythematosus. J Immunol Res, 2014;2014:809389.

[16] Li M, Zhang W, Leng X, et al. Chinese SLE Treatment and Research Group (CSTAR) Registry: I. Major Clinical Characteristics of Chinese Patients with Systemic Lupus Erythematosus. Lupus, 2013;22(11):1192-1199.

[17] Gladman DD, Urowitz MB, Goldsmith C, et al. The Development and Initial Validation of the Systemic Lupus International Collaborating Clinics/American College of Rheumatology Damge Index in Patients with Systemic Lupus Erythematosus. Arthritis Rheum, 1996;39(3):363-369.

[18] Gladman DD, Urowitz MB, Goldsmith CH. The Reliability of the Systemic Lupus International Collaborating Clinics/American College of Rheumatology Damge Index in Patients with Systemic Lupus Erythematosus. Arthritis Rheum, 1997;40(5):809-813.

[19] Petri M, Orbai AM, Alarcon GS, et al. Derivation and Validation of the Systemic Lupus International Collaborating Clinics Classification Criteria for Systemic Lupus Erythematosus. Arthritis Rheum, 2012;64(8):2677-2686.

[20] Aringer M, Costenbader K, Daikh D, et al. 2019 European League Against Rheumatism/American College of Rheumatology Classification Criteria for Systemic Lupus Erythematosus. Arthritis Rheumatol, 2019;71(9):1400-1412.

[21] Funauchi M, Shimadzu H, Tamaki C, et al. Survival Study by Organ Disorders in 306 Japanese Patients with Systemic Lupus Erythematosus: Results from a Single Center. Rheumatol Int, 2007;27(3):243-249.

[22] Tokano $\mathrm{Y}$, Morimoto $\mathrm{S}$, Amano $\mathrm{H}$, et al. The Relationship Between Initial Clinical Manifestation and Long-term Prognosis of Patients with Systemic Lupus Erythematosus. Modern Rheumatol, 
2005;15(4):275-282.

[23] Peng L, Wang Z, Li M, et al. Flares in Chinese Systemic Lupus Erythematosus Patients: A 6-Year Follow-up Study. Clin Rheumatol, 2017;36(12):2727-2732.

[24] Mao S, Shen H, Zhang J. Systemic Lupus Erythematosus and Malignancies Risk. J Cancer Res Clin Oncol, 2016;142(1):253-262.

[25] Cao L, Tong H, Xu G, et al. Systemic Lupus Erythematous and Malignancy Risk: A Meta-analysis. PloS one, 2015;10(4):e0122964.

[26] Wang Z, Li M, Wang Y, et al. Long-term Mortality and Morbidity of Patients with Systemic Lupus Erythematosus: A Single-center Cohort Study in China. Lupus, 2018;27(5):864-869.

[27] Zhao J, Bai W, Zhu P, et al. Chinese SLE Treatment and Research Group (CSTAR) Registry VII: Prevalence and Clinical Significance of Serositis in Chinese Patients with Systemic Lupus Erythematosus. Lupus, 2016;25(6):652-657.

[28] Appenzeller S, Pereira DA, Costallat LT. Greater Accrual Damage in Late-onset Systemic Lupus Erythematosus: A Long-term Follow-up Study. Lupus, 2008;17(11):1023-1028.

[29] Kim H, Levy DM, Silverman ED, et al. A Comparison Between Childhood and Adult Onset Systemic Lupus Erythematosus Adjusted for Ethnicity from the 1000 Canadian Faces of Lupus Cohort. Rheumatology, 2019;58(8):1393-1399.

[30] Mikdashi J, Handwerger B. Predictors of Neuropsychiatric Damage in Systemic Lupus Erythematosus: Data From the Maryland Lupus Cohort. Rheumatology, 2004;43(12):1555-1560.

[31] Higuera-Ortiz V, Mora-Arias T, Castillo-Martinez D, et al. Anti-Ro/ SSAAntibodies are Associated with Severe Mitral Valve Regurgitation in Patients with Systemic Lupus Erythematosus. Modern Rheumatol, 2017;27(3):476-480.

[32] Sutton EJ, Davidson JE, Bruce IN. The Systemic Lupus International Collaborating Clinics (SLICC) Damage Index: A Systematic Literature Review. Semin Arthritis Rheum, 2013;43(3):352-361.

[33] Cardoso CR, Signorelli FV, Papi JA, et al. Initial and Accrued Damage as Predictors of Mortality in Brazilian Patients with Systemic Lupus Erythematosus: A Cohort Study. Lupus, 2008;17(11): 1042-1048.

[34] Conti F, Ceccarelli F, Perricone C, et al. The Chronic Damage in Systemic Lupus Erythematosus is Driven by Flares, Glucocorticoids and Antiphospholipid Antibodies: Results from a Monocentric Cohort. Lupus, 2016;25(7):719-726. 